\section{Burden of Imported Cases of Infection or Colonization With Multidrug-Resistant Organisms in a German University Hospital}

To the Editor-The emergence and spread of multidrug-resistant organisms (MDROs) presents a major public health threat in Germany and worldwide. ${ }^{1}$ In particular, we are referring to methicillin-resistant Staphylococcus aureus (MRSA), vancomycin-resistant enterococci (VRE), and extended-spectrum $\beta$-lactamase (ESBL)-producing Enterobacteriaceae. Prevention strategies include screening programs for the early detection of MDROs, decontamination strategies for the sustainable reduction in MRSA carriage, improved compliance with hand hygiene to decrease the transmission of MDROs, and reduction in antibiotic selection pressure by the implementation of policies to control the use of antibiotics. ${ }^{2-4}$ It is generally agreed that antibiotic selection pressure and transmission are the 2 main reasons for the emergence and spread of MDROs.

Since midyear 2006, the University Medical Center Freiburg has faced a dramatic increase in patients infected with ESBL-producing organisms. Some of these patients (eg, pregnant women) had no risk factors, such as antibiotic exposure or hospital stays, in their past history. This brought us to the hypothesis that there might be an important reservoir of ESBL-producing Enterobacteriaceae or, generally, MDROs in the community. The aim of our study was to analyze the burden of imported cases of MDRO infection or colonization in the hospital and to discuss the consequences.
The University Medical Center Freiburg is a 1,600-bed university hospital with all the clinical specialities. Approximately 60,000 patients are admitted each year, accounting for total of 440,000 patient-days. Generally, besides standard precautions, barrier precautions are recommended at the University Medical Center Freiburg for all patients colonized or infected with MRSA and/or VRE, as well as all patients infected with ESBL-producing bacteria. Barrier precautions include the use of a single room or cohorting with other colonized or infected patients. Staff members wear gloves and gowns, and roommates undergo screening.

In our study, a case was defined as the detection of an MDRO in a patient. Every patient was counted only once during a hospital stay. Readmission within 8 days of discharge was not counted as a new case. Cases were defined as nosocomial if an MDRO was detected in a clinical or screening culture of a sample obtained 48 hours after hospital admission, irrespective of whether admission screening was performed or not. Admission screening for MRSA in high-risk patients has been recommended since 2004. High-risk patients are defined as patients who have chronic open wounds, are undergoing dialysis, have been transferred from hospitals or institutions with a known MRSA problem, and/or, additionally, are older than 60 years of age and have been admitted to an intensive care unit. An electronic database of patients who had been infected or colonized with an MDRO in the past has been in place since 2005. All these patients were screened at hospital readmission. If a patient was found to have a culture positive for an MDRO at readmission or if an MDRO was detected in a sample obtained within 48 hours after hospital admission, the case was defined as imported to

TA B LE. Prevalence of Cases of Detection of Multidrug-Resistant Organism (MDRO) Infection or Colonization and of Cases of Infection and Bloodstream Infection (BSI) Caused by MDROs, at Freiburg University Hospital, 2007

\begin{tabular}{|c|c|c|c|c|c|c|c|c|}
\hline \multirow[b]{2}{*}{ Type of case, origin } & \multicolumn{2}{|c|}{ MRSA } & \multicolumn{2}{|c|}{ VRE } & \multicolumn{2}{|c|}{$\begin{array}{l}\text { ESBL-producing } \\
\text { organism }\end{array}$} & \multicolumn{2}{|c|}{ All MDROs } \\
\hline & $\begin{array}{c}\text { No. of cases } \\
\text { per } 1,000 \\
\text { patient-days }\end{array}$ & $\begin{array}{l}\text { Total no. } \\
\text { of cases }\end{array}$ & $\begin{array}{c}\text { No. of cases } \\
\text { per } 1,000 \\
\text { patient-days }\end{array}$ & $\begin{array}{l}\text { Total no. } \\
\text { of cases }\end{array}$ & $\begin{array}{c}\text { No. of cases } \\
\text { per } 1,000 \\
\text { patient-days }\end{array}$ & $\begin{array}{l}\text { Total no. } \\
\text { of cases }\end{array}$ & $\begin{array}{l}\text { No. of cases } \\
\text { per } 1,000 \\
\text { patient-days }\end{array}$ & $\begin{array}{l}\text { Total no. } \\
\text { of cases }\end{array}$ \\
\hline \multicolumn{9}{|l|}{ Detection of $\mathrm{MDRO}^{\mathrm{a}}$} \\
\hline Imported & 0.47 & 204 & 0.09 & 37 & 0.17 & 73 & 0.73 & 314 \\
\hline \multicolumn{9}{|l|}{ MDRO infection } \\
\hline Imported & 0.10 & 44 & 0.00 & 2 & 0.16 & 62 & 0.26 & 108 \\
\hline Nosocomial & 0.05 & 22 & 0.01 & 6 & 0.10 & 47 & 0.16 & 85 \\
\hline \multicolumn{9}{|l|}{ MDRO BSI } \\
\hline Imported & 0.014 & 6 & 0.002 & 1 & 0.018 & 8 & 0.022 & 15 \\
\hline Nosocomial & 0.002 & 1 & 0.002 & 1 & 0.018 & 8 & 0.022 & 10 \\
\hline
\end{tabular}

NOTE. BSI, bloodstream infection; ESBL, extended-spectrum $\beta$-lactamase; MRSA, methicillin-resistant Staphylococcus aureus, VRE, vancomycin-resistant enterococci.

anfection or colonization. 
the hospital. All cases from the electronic database were included in the analysis.

In our study, the prevalence of cases of imported MDRO infection or colonization was higher than the prevalence of cases of nosocomial MDRO infection or colonization (Table). This was also true for cases of MRSA infection and bloodstream infection. During the last 2 years, the incidence density of imported cases of MRSA infection or colonization have increased from 0.42 to 0.47 cases per 1,000 patient-days, and cases of imported colonization or infection with MRSA and with ESBL-producing organisms have far outweighed nosocomial cases.

Analyzing our data, we were surprised at the magnitude of the burden of cases of imported MDRO infection or colonization, even more so because antibiotic selection pressure and transmission in the hospital are generally agreed to be the 2 main reasons for the emergence and spread of MDROs. However, benchmarking data provided by the the MRSAKISS module of the German national infection surveillance system (Krankenhaus Infektions Surveillance System [KISS]) show that, in $2006,70 \%$ of all cases of MRSA infection or colonization were imported to 133 participating hospitals and that only $30 \%$ of the cases were nosocomial, which corresponds to a median incidence density of 0.21 cases of nosocomial MRSA infection or colonization per 1,000 patientdays. ${ }^{5}$ In addition, Trautmann et al..$^{6}$ showed that, after an intensified MRSA infection-control program that included the screening of high-risk patients, the number of cases of imported MRSA infection or colonization steadily increased after 2005. Therefore, we hypothesize that the burden of cases of imported MDRO infection or colonization is considerably higher than the burden of cases of nosocomial MDRO infection or colonization if screening policies, as recommended by the Robert Koch Institute in Germany, and warning systems are in place. Furthermore, the number of cases of MDRO infection or colonization imported to the hospital can be expected to be even higher, because no general screening for ESBL-producing organisms or VRE was established. Therefore, all cases detected in culture samples obtained 48 hours after admission were defined as nosocomial even if they might have been present at admission but were not detected because of a lack of screening.

The dramatic increase in the incidence of colonization or infection with ESBL-producing strains of Enterobacteriaceae in hospitals, which was accompanied by an increase in the incidence in the community, suggests that the community could act as a reservoir and that food could contribute to the spread of these strains. ${ }^{7}$ Furthermore, a recent study $y^{8}$ showed that soil bacteria subsisting on antibiotics were phylogenetically diverse, many closely related to human pathogens and resistant to multiple antibiotics at clinically relevant concentrations. The authors of that study suggest that this unappreciated reservoir of antibiotic resistance determinants can contribute to the increasing levels of multiple antibiotic resistance in pathogenic bacteria. ${ }^{8}$

Although ours was a single-center study, we came to the conclusion that research into the reservoir of patients with MDRO infection or colonization should be intensified and should especially include outpatients. As long as efforts concentrate only on the detection of MDRO infection or colonization in the hospital and on the prevention of transmission in medical facilities, the number of cases can be, at most, kept at the same level. Therefore, to improve MDRO infection control programs, it might not be sufficient to restrict interventions and decontamination strategies to high-risk areas; rather, the whole the community should be included.

\section{ACKNOWLEDGMENTS}

Potential conflicts of interest. All authors report no conflicts of interest relevant to this article.

Elisabeth Meyer, MD; Andreas Conrad, MD;
Christian Schneider, MD; Annerose Serr, MD;
Regina Babikir; Markus Dettenkofer, MD, PhD

From the Institute of Environmental Health Sciences (E.M., A.C., R.B., M.D.) and the Department for Medical Microbiology and Hygiene (C.S., A.S.), University Medical Center Freiburg, Freiburg, Germany.

Address reprint requests to Elisabeth Meyer, MD, Institute of Hygiene and Environmental Medicine, Charité University Medicine Berlin, Hindenburgdamm 12, 12203 Berlin, Germany (elisabeth.meyer@charite.de). Infect Control Hosp Epidemiol 2008; 29:1195-1196

(C) 2008 by The Society for Healthcare Epidemiology of America. All rights reserved. 0899-823X/2008/2912-0018\$15.00. DOI: 10.1086/592409

\section{REFERENCES}

1. European Antimicrobial Resistance Surveillance System (EARSS). EARSS Annual Report 2006. Available at: http://www.rivm.nl/earss/Images/ EARSS\%202006\%20Def_tcm61-44176.pdf. Accessed June 20, 2007.

2. Harbarth S. Control of endemic methicillin-resistant Staphylococcus aureus - recent advances and future challenges. Clin Microbiol Infect 2006; 12: 1154-1162.

3. Siegel JD, Rhinehart E, Jackson M, Chiarello L. 2007 Guideline for isolation precautions: preventing transmission of infectious agents in health care settings. Am J Infect Control 2007; 35:\$65-S164.

4. Siegel JD, Rhinehart E, Jackson M, Chiarello L. Management of multidrugresistant organisms in health care settings, 2006. Am J Infect Control 2007; 35:S165-S193.

5. Chaberny IF, Sohr D, Ruden H, Gastmeier P. Development of a surveillance system for methicillin-resistant Staphylococcus aureus in German hospitals. Infect Control Hosp Epidemiol 2007; 28:446-452.

6. Trautmann M, Pollitt A, Loh $U$, et al. Implementation of an intensified infection control program to reduce MRSA transmissions in a German tertiary care hospital. Am J Infect Control 2007; 35:643-649.

7. Mesa RJ, Blanc V, Blanch AR, et al. Extended-spectrum $\beta$-lactamaseproducing Enterobacteriaceae in different environments (humans, food, animal farms and sewage). J Antimicrob Chemother 2006; 58:211-215.

8. Dantas G, Sommer MO, Oluwasegun RD, Church GM. Bacteria subsisting on antibiotics. Science 2008; 320:100-103. 